\title{
A Proof Strategy Language and Proof Script Generation for Isabelle/HOL
}

\author{
Yutaka Nagashima and Ramana Kumar \\ Data61, CSIRO / UNSW
}

\begin{abstract}
We introduce a language, PSL, designed to capture high level proof strategies in Isabelle/HOL. Given a strategy and a proof obligation, PSL's runtime system generates and combines various tactics to explore a large search space with low memory usage. Upon success, PSL generates an efficient proof script, which bypasses a large part of the proof search. We also present PSL's monadic interpreter to show that the underlying idea of PSL is transferable to other ITPs.
\end{abstract}

\section{Introduction}

Currently, users of interactive theorem provers (ITPs) spend too much time iteratively interacting with their ITP to manually specialise and combine tactics as depicted in Fig. 1a. This time consuming process requires expertise in the ITP, making ITPs more esoteric than they should be. The integration of powerful automated theorem provers (ATPs) into ITPs ameliorates this problem significantly; however, the exclusive reliance on general purpose ATPs makes it hard to exploit users' domain specific knowledge, leading to combinatorial explosion even for conceptually straight-forward conjectures.

To address this problem, we introduce PSL, a programmable, extensible, meta-tool based framework, to Isabelle/HOL [21. We provide PSL (available on GitHub [17) as a language, so that its users can encode proof strategies, abstract

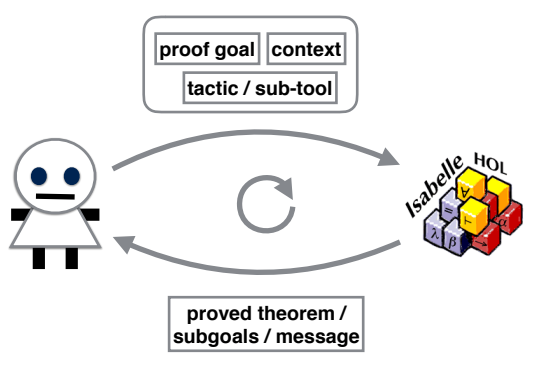

(a) Standard proof attempt

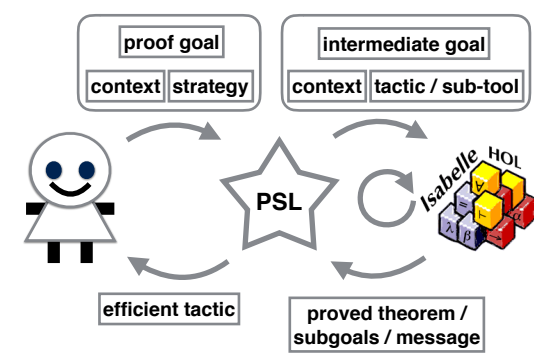

(b) Proof attempt with PSL

Fig. 1: Comparison of proof development processes 
descriptions of how to attack proof obligations, based on their intuitions about a conjecture. When applied to a proof obligation, PSL's runtime system creates and combines several tactics based on the given proof strategy. This makes it possible to explore a larger search space than has previously been possible with conventional tactic languages, while utilising users' intuitions on the conjecture.

We developed PSL to utilise engineers' downtime: with PSL, we can run an automatic proof search for hours while we are attending meetings, sleeping, or reviewing papers. PSL makes such expensive proof search possible on machines with limited memory: PSL's runtime truncates failed proof attempts as soon as it backtracks to minimise its memory usage.

Furthermore, PSL's runtime system attempts to generate efficient proof scripts from a given strategy by searching for the appropriate specialisation and combination of tactics for a particular conjecture without direct user interaction, as illustrated in Fig. 1 $1 \mathrm{~b}$. Thus, PSL not only reduces the initial labour cost of theorem proving, but also keeps proof scripts interactive and maintainable by reducing the execution time of subsequent proof checking.

In Isabelle, sledgehammer adopts a similar approach [2. It exports a proof goal to various external ATPs and waits for them to find a proof. If the external provers find a proof, sledgehammer tries to reconstruct an efficient proof script in Isabelle using hints from the ATPs. sledgehammer is often more capable than most tactics but suffers from discrepancies between the different provers and logics used. While we integrated sledgehammer as a sub-tool in PSL, PSL conducts a search using Isabelle tactics, thus avoiding the problems arising from the discrepancies and proof reconstruction.

The underlying implementation idea in PSL is the monadic interpretation of proof strategies, which we introduce in Section 6 . We expect this prover-agnostic formalization brings the following strengths of PSL to other ITPs such as Lean [15] and Coq [27:

- runtime tactic generation based on user-defined procedures,

- memory-efficient large-scale proof search, and

- efficient-proof-script generation for proof maintenance.

\section{Background}

Interactive theorem proving can be seen as the exploration of a search tree. Nodes of the tree represent proof states. Edges represent applications of tactics, which transform the proof state. The behaviour of tactics are, in general, not completely predictable since they are context sensitive: they behave differently depending on the information stored in background proof contexts. A proof context contains the information such as constants defined so far and auxiliary lemmas proved prior to the step. Therefore, the shape of the search tree is not known in advance.

The goal is to find a node representing a solved state: one in which the proof is complete. The search tree may be infinitely wide and deep, because there are endless variations of tactics that may be tried at any point. The goal for a PSL 


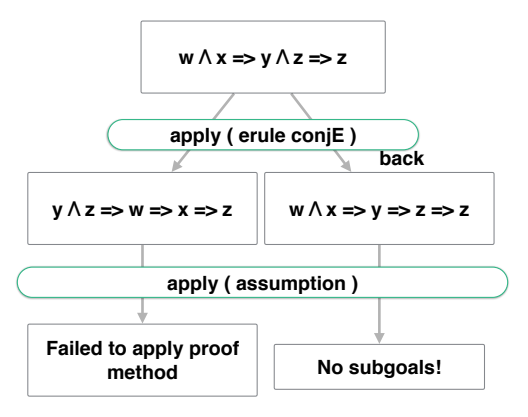

(a) External view

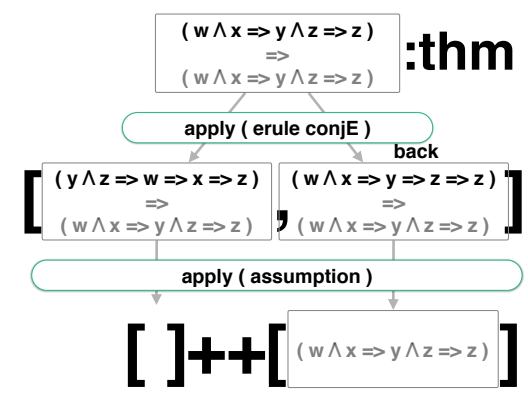

(b) Internal view

Fig. 2: External and internal view of proof search tree.

strategy is to direct an automated search of this tree to find a solved state; PSL will reconstruct an efficient path to this state as a human-readable proof script.

Fig. 2a shows an example of proof search. At the top, the tactic erule conjE is applied to the proof obligation $\mathrm{w} \wedge \mathrm{x} \Rightarrow \mathrm{y} \wedge \mathrm{z} \Rightarrow \mathrm{z}$. This tactic invocation produces two results, as there are two places to apply conjunction elimination. Applying conjunction elimination to $\mathrm{w} \wedge \mathrm{x}$ returns the first result, while doing so to $\mathrm{y} \wedge \mathrm{z}$ produces the second result. Subsequent application of proof by assumption can discharge the second result; however, assumption does not discharge the first one since the $\mathrm{z}$ in the assumptions is still hidden by the conjunction. Isabelle's proof language, Isar, returns the first result by default, but users can access the subsequent results using the keyword back.

Isabelle represents this non-deterministic behaviour of tactics using lazy sequences: tactics are functions of type thm $\rightarrow[\mathrm{thm}]$, where $[\cdot]$ denotes a (possibly infinite) lazy sequence [24]. Fig. 2b illustrates how Isabelle internally handles the above example. Each proof state is expressed as a (possibly nested) implication which assumes proof obligations to conclude the conjecture. One may complete a proof by removing these assumptions using tactics. Tactic failure is represented as an empty sequence, which enables backtracking search by combining multiple tactics in a row [29]. For example, one can write apply (erule conjE, assumption) using the sequential combinator , (comma) in Isar; this tactic traverses the tree using backtracking and discharges the proof obligation without relying on the keyword back.

The search tree grows wider when choosing between multiple tactics, and it grows deeper when tactics are combined sequentially. In the implementation language level, the tactic combinators in Isabelle include THEN for sequential composition (corresponding to , in Isar), APPEND for non-deterministic choice, ORELSE for deterministic choice, and REPEAT for iteration.

Isabelle/HOL comes with several default tactics such as auto, simp, induct, rule, and erule. When using tactics, proof authors often have to adjust tactics using modifiers for each proof obligation. succeed and fail are special tactics: 
succeed takes a value of type thm, wraps it in a lazy sequence, and returns it without modifying the value. fail always returns an empty sequence.

\section{Syntax of PSL}

The following is the syntax of PSL. We made PSL's syntax similar to that of Isabelle's tactic language aiming at the better usability for users who are familiar with Isabelle's tactic language.

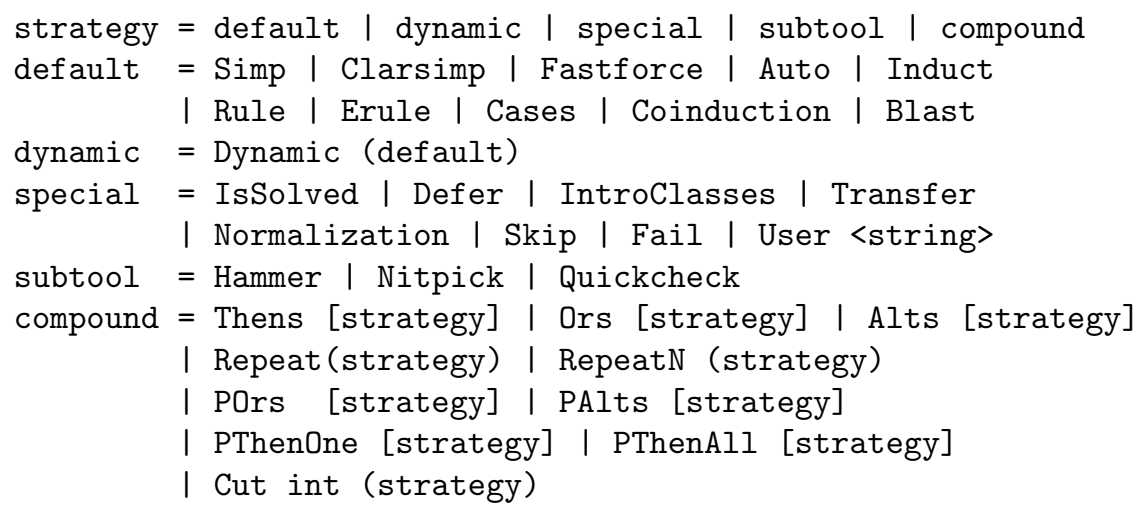

The default strategies correspond to Isabelle's default tactics without arguments, while dynamic strategies correspond to Isabelle's default tactics that are specialised for each conjecture. Given a dynamic strategy and conjecture, the runtime system generates variants of the corresponding Isabelle tactic. Each of these variants is specialised for the conjecture with a different combination of promising arguments found in the conjecture and its proof context. It is the purpose of the PSL runtime system to select the right combination automatically.

subtool represents Isabelle tools such as sledgehammer [2] and counterexample finders. The compound strategies capture the notion of tactic combinators: Thens corresponds to THEN, Ors to ORELSE, Alts to APPEND, and Repeat to REPEAT. POrs and PAlts are similar to Ors and Alts, respectively, but they admit parallel execution of sub-strategies. PThenOne and PThenAll take exactly two sub-strategies, combine them sequentially and apply the second sub-strategy to the results of the first sub-strategy in parallel in case the first sub-strategy returns multiple results. Contrary to PThenAll, PThenOne stops its execution as soon as it produces one result from the second sub-strategy. Users can integrate user-defined tactics, including those written in Eisbach [14], into PSL strategies using User. Cut limits the degree of non-determinism within a strategy.

In the following, we explain how to write strategies and how PSL's runtime system interprets strategies with examples.

\section{PSL by Example}

Example 1. For our first example, we take the following lemma about depth-first search from an entry [22]. 


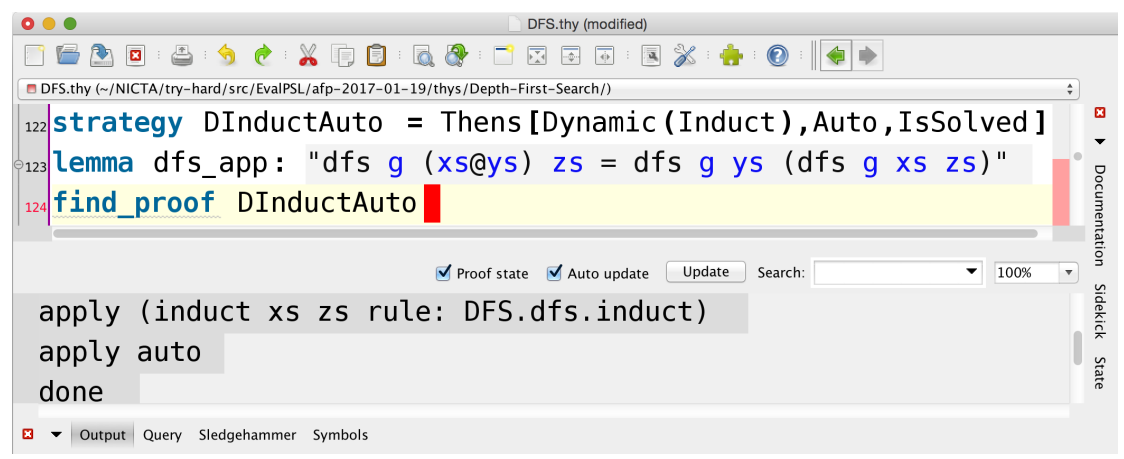

Fig. 3: Screenshot for Example 1.

lemma dfs_app: "dfs $g(x s$ @ $y s)$ zs = dfs $g$ ys (dfs $g$ xs $z s) "$

where dfs is a recursively defined function for depth-first search. As dfs is defined recursively, it is natural to expect that its proof involves some sort of mathematical induction. However, we do not know exactly how we should conduct mathematical induction here; therefore, we describe this rough idea as a proof strategy, DInductAuto, with the keyword strategy, and apply it to dfs_app with the keyword find_proof as depicted in Fig. 3. Invoked by find_proof, PSL's runtime system interprets DInductAuto. For example, it interprets Auto as Isabelle's default tactic, auto.

The interpretation of Dynamic (Induct) is more involved: the runtime generates tactics using the information in $\mathrm{df} \mathbf{s}_{\text {_app }}$ and its background context. First, PSL collects the free variables (noted in italics above) in dfs_app and applicable induction rules stored in the context. PSL uses the set of free variables to specify two things: on which variables instantiated tactics conduct mathematical induction, and which variables should be generalised in the induction scheme. The set of applicable rules are used to specify which rules to use. Second, PSL creates the powerset out of the set of all possible modifiers. Then, it attempts to instantiate a variant of the induct tactic for each subset of modifiers. Finally, it combines all the variants of induct with unique results using APPEND. In this case, PSL tries to generate 4160 induct tactics for dfs_app by passing several combinations of modifiers to Isabelle; however, Isabelle cannot produce valid induction schemes for some combinations, and some combinations lead to the same induction scheme. The runtime removes these, focusing on the 223 unique results. PSL's runtime combine these tactics with auto using THEN.

PSL's runtime interprets IsSolved as the is_solved tactic, which checks if any proof obligations are left or not. If obligations are left, is_solved behaves as fail, triggering backtracking. If not, is_solved behaves as succeed, allowing the runtime to stop the search. This is how DInductAuto uses IsSolved to ensure that no sub-goals are left before returning an efficient proof script. For 
dfs_app, PSL interprets DInductAuto as the following tactic:

\section{(induct1 APPEND induct2 APPEND...) THEN auto THEN is_solved}

where induct_ns are variants of the induct tactic specialised with modifiers.

Within the runtime system, Isabelle first applies induct1 to dfs_app, then auto to the resultant proof obligations. Note that each induct tactic and auto is deterministic: it either fails or returns a lazy sequence with a single element. However, combined together with APPEND, the numerous variations of induct tactics en mass are non-deterministic: if is_solved finds remaining proof obligations, Isabelle backtracks to the next induct tactic, induct2 and repeats this process until either it discharges all proof obligations or runs out of the variations of induct tactics. The numerous variants of induct tactics from DInductAuto allow Isabelle to explore a larger search space than its naive alternative, induct THEN auto, does. Fig. 4a illustrates this search procedure. Each edge and curved square represents a tactic application and a proof state, respectively, and edges leading to no object stand for tactic failures. The dashed objects represent possible future search space, which PSL avoids traversing by using lazy sequences.

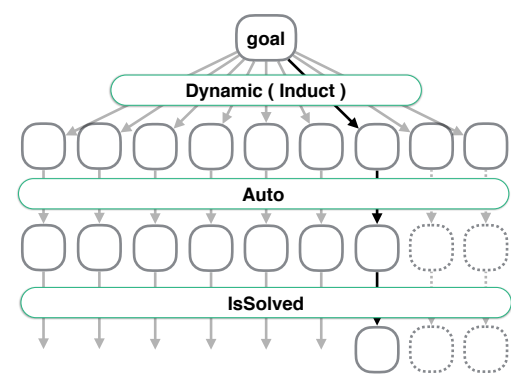

(a) DInductAuto

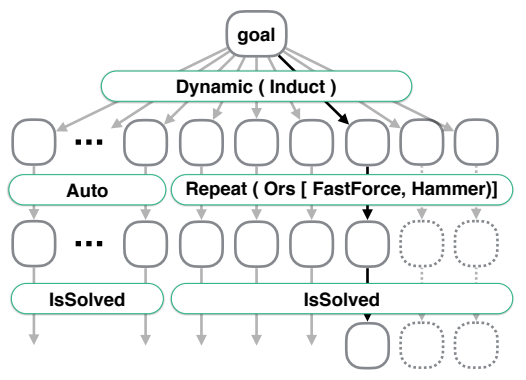

(b) some_induct2

Fig. 4: Proof search tree for some_induct

The larger search space specified by DInductAuto leads to a longer search time. PSL addresses this performance problem by tracing Isabelle's proof search: it keeps a log of successful proof attempts while removing backtracked proof attempts. The monadic interpretation discussed in Section 6 let PSL remove failed proof steps as soon as it backtracks. This minimises PSL memory usage, making it applicable to hours of expensive automatic proof search. Furthermore, since PSL follows Isabelle's execution model based on lazy sequences, it stops proof search as soon as it finds a specialisation and combination of tactics, with which Isabelle can pass the no-proof-obligation test imposed by is_solved.

We still need a longer search time with PSL, but only once: upon success, PSL converts the log of successful attempts into an efficient proof script, which 
bypasses a large part of proof search. For dfs_app, PSL generates the following proof script from DInductAuto.

apply (induct xs zs rule: DFS.dfs.induct) apply auto done

We implemented PSL as an Isabelle theory; to use it, PSL users only have to import the relevant theory files to use PSL to their files. Moreover, we have integrated PSL into Isabelle/Isar, Isabelle's proof language, and Isabelle/jEdit, its standard editor. This allows users to define and invoke their own proof strategies inside their ongoing proof attempts, as shown in Figure 3, and if the proof search succeeds PSL presents a proof script in jEdit's output panel, which users can copy to the right location with one click. All generated proof scripts are independent of PSL, so users can maintain them without PSL.

Example 2. DInductAuto is able to pick up the right induction scheme for relatively simple proof obligations using backtracking search. However, in some cases even if PSL picks the right induction scheme, auto fails to discharge the emerging sub-goals. In the following, we define InductHard, a more powerful strategy based on mathematical induction, by combining Dynamic (Induct) with more involved sub-strategies to utilise external theorem provers.

strategy SolveAllG = Thens [Repeat(Ors [Fastforce, Hammer]), IsSolved]

strategy PInductHard = PThenOne [Dynamic (Induct), SolveAllG]

strategy InductHard = Ors [DInductAuto, PInductHard]

PSL's runtime system interprets Fastforce and Hammer as the fastforce tactic and sledgehammer, respectively. Both fastforce and sledgehammer try to discharge the first sub-goal only and return an empty sequence if they cannot discharge the sub-goal.

The repetitive application of sledgehammer would be very time consuming. We mitigate this problem using Ors and PThenOne. Combined with Ors, PSL executes PInductHard only if DInductAuto fails. When PInductHard is called, it first applies Dynamic (Induct), producing various induction schemes and multiple results. Then, SolveAllG tries to discharge these results in parallel. The runtime stops its execution when SolveAllG returns at least one result representing a solved state. We apply this strategy to the following conjecture, which states the two versions of depth first search programs ( $d f s 2$ and $d f s$ ) return the same results given the same inputs.

lemma "dfs2 g xs ys = dfs $g$ xs ys"

Then, our machine with 28 cores returns the following script within 3 minutes:

apply (induct xs ys rule: DFS.dfs2.induct)

apply fastforce apply (simp add: dfs_app) done

Fig. $4 \mathrm{~b}$ roughly shows how the runtime system found this proof script. The runtime first tried to find a complete proof as in Example 1, but without much 
success. Then, it interpreted PInductHard. While doing so, it found that induction on $x s$ and $y s$ using DFS.dfs2.induct leads to two sub-goals both of which can be discharged either by fastforce or sledgehammer. For the second sub-goal, sledgehammer found out that the result of Example 1 can be used as an auxiliary lemma to prove this conjecture. Then, it returns an efficient-proofscript (simp add: dfs_app) to PSL, before PSL combines this with other parts and prints the complete proof script.

Example 3. In the previous examples, we used IsSolved to get a complete proof script from PSL. In Example 3, we show how to generate incomplete but useful proof scripts, using Defer. Incomplete proofs are specially useful when ITP users face numerous proof obligations, many of which are within the reach of high-level proof automation tools, such as sledgehammer, but a few of which are not.

Without PSL, Isabelle users had to manually invoke sledgehammer several times to find out which proof obligations sledgehammer can discharge. We developed a strategy, HamCheck, to automate this time-consuming process. The following shows its definition and a use case simplified for illustrative purposes.

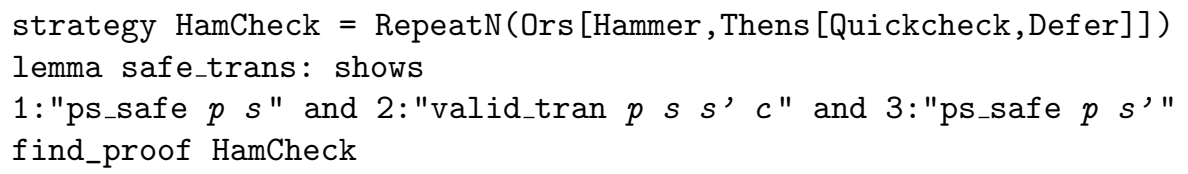

We made this example simple, so that two sub-goals, 1:"ps_safe $p s$ " and 3: "ps_safe $p s^{\prime}$ ", are not hard to prove; however, they are still beyond the scope of commonly used tactics, such as fastforce.

Generally, for a conjecture and a strategy of the form of RepeatN (strategy), PSL applies strategy to the conjecture as many times as the number of proof obligations in the conjecture. In this case, PSL applies Ors [Hammer, Thens [Quickcheck, Defer]] to safe_trans three times.

Note that we integrated quickcheck and nitpick into PSL as assertion tactics. Assertion tactics provide mechanisms for controlling proof search based on a condition: such a tactic takes a proof state, tests an assertion on it, then behaves as succeed or fail accordingly. We have already seen one of them in the previous examples: is_solved.

Ors [Hammer, Thens [Quickcheck, Defer]] first applies sledgehammer. If sledgehammer does not find a proof, it tries to find counter-examples for the sub-goal using quickcheck. If quickcheck finds no counter-examples, PSL interprets Defer as defer_tac 1, which postpones the current sub-goal to the end of the list of proof obligations.

In this example, sledgehammer fails to discharge 2: "valid_tran $p s s^{\text {, }}$ $c$ ". When sledgehammer fails, PSL passes 2 to Thens [Quickcheck, Defer], which finds no counter-example to 2 and sends 2 to the end of the list; then, PSL continues working on the sub-goal 3 with sledgehammer. The runtime stops its execution after applying Ors [Hammers, Thens [Quickcheck, Defer]] three times, generating the following proof script. This script discharges 1 and 3, but 
Table 1: The number of automatically proved proof obligations from assignments.

\begin{tabular}{cccccccccccc}
\hline assignments $[1]$ & ass_1 & ass_2 & ass_3 & ass_4 & ass_5 & ass_6 & ass_8 & ass_9 & ass_11 & sum \\
POs & 19 & 22 & 52 & 82 & 64 & 26 & 52 & 61 & 26 & 404 \\
\hline TH 30s & 17 & 21 & 30 & 66 & 36 & 11 & 36 & 31 & 14 & 262 \\
SH 30s & $14(13)$ & 5 & 27 & 61 & $41(39)$ & $12(11)$ & $45(39)$ & $32(30)$ & 15 & $252(241)$ \\
TH $\backslash$ SH 30s & 4 & 16 & 8 & 10 & 6 & 2 & 1 & 6 & 1 & 54 \\
TH 300s & 18 & 22 & 35 & 71 & 55 & 14 & 40 & 35 & 20 & 310 \\
SH 300s & $14(13)$ & 5 & 27 & 61 & $44(42)$ & $13(12)$ & $46(39)$ & $32(30)$ & 17 & $259(246)$ \\
TH\SH 300s & 5 & 17 & 10 & 10 & 17 & 3 & 0 & 6 & 3 & 71 \\
\hline
\end{tabular}

Table 2: The number of automatically proved proof obligations from exercises.

\begin{tabular}{|c|c|c|c|c|c|c|c|c|c|c|c|c|c|c|c|c|}
\hline \multicolumn{3}{|c|}{ exercise 1] e1 e2 } & e3 & \multicolumn{9}{|c|}{ e4 e5a e5b e6 e7a e7b e8a e8b e9 } & \multicolumn{3}{|c|}{ e10 e11 e12 } & \multirow{2}{*}{$\begin{array}{c}\text { sum } \\
321\end{array}$} \\
\hline POs & 15 & 7 & 42 & 23 & 13 & 83 & 4 & 3 & 9 & 10 & 26 & 31 & 15 & 10 & 30 & \\
\hline TH $30 \mathrm{~s}$ & 12 & 4 & 27 & 11 & 9 & 65 & 1 & 0 & 5 & 7 & 11 & 14 & 5 & 4 & 8 & 183 \\
\hline SH 30 s & 8 & 3 & $26(25)$ & 15 & 11 & 74 & 2 & 0 & 6 & 7 & 9 & 17 & $5(4)$ & 6 & 10 & $199(197)$ \\
\hline $\mathrm{TH} \backslash \mathrm{SH} 30 \mathrm{~s}$ & 4 & 2 & 5 & 0 & 0 & 1 & 0 & 0 & 1 & 1 & 4 & 3 & 1 & 0 & 1 & 23 \\
\hline TH $300 \mathrm{~s}$ & 12 & 5 & 29 & 17 & 11 & 74 & 1 & 0 & 8 & 7 & 12 & 19 & 6 & 9 & 12 & 222 \\
\hline $\mathrm{SH} 300 \mathrm{~s}$ & 8 & 3 & $27(26)$ & 15 & 11 & 74 & 3 & 0 & 6 & 7 & 12 & & $6(5)$ & 6 & 10 & $205(203)$ \\
\hline $\mathrm{TH} \backslash \mathrm{SH} 300 \mathrm{~s}$ & 4 & 2 & 5 & 2 & 0 & 1 & 0 & 0 & 2 & 1 & 2 & 3 & 1 & 3 & 3 & 29 \\
\hline
\end{tabular}

it leaves 2 as the meaningful task for human engineers, while assuring there is no obvious counter-examples for 2 .

apply (simp add: state_safety ps_safe_def)

defer apply (simp add: state_safety ps_safe_def)

\section{The default strategy: try_hard.}

PSL comes with a default strategy, try_hard. Users can apply try hard as a completely automatic tool: engineers need not provide their intuitions by writing strategies. Unlike other user-defined strategies, one can invoke this strategy by simply typing try_hard without find_proof inside a proof attempt. The lack of input from human engineers makes try hard less specific to each conjecture; however, we made try_hard more powerful than existing proof automation tools for Isabelle by specifying larger search spaces presented in Appendix A.

We conducted a judgement-day style evaluation [3] of try_hard against selected theory files from the Archive of Formal Proofs (AFP), coursework assignments and exercises 1, and Isabelle's standard library. Table 1, 2 and $3^{1}$ show

\footnotetext{
${ }^{1} \mathrm{TH}$ and $\mathrm{SH}$ stand for the number of obligations discharged by try_hard and
} sledgehammer, respectively. $\mathrm{TH} \backslash \mathrm{SH}$ represents the number of goals to which try hard 
Table 3: The number of automatically proved proof goals from AFP entries and Isabelle's standard libraries.

\begin{tabular}{cccccccc}
\hline theory name & POs & TH & SH & TH $\backslash$ SH & TH & SH & TH $\backslash \mathrm{SH}$ \\
\hline time out & - & $30 \mathrm{~s}$ & $30 \mathrm{~s}$ & $30 \mathrm{~s}$ & $300 \mathrm{~s}$ & $300 \mathrm{~s}$ & $300 \mathrm{~s}$ \\
\hline DFS.thy [22] & 51 & 24 & 28 & 6 & 34 & 29 & 7 \\
Efficient_Sort.thy [26] & 75 & 27 & $28(26)$ & 8 & 33 & $31(28)$ & 9 \\
List_Index.thy [19] & 105 & 48 & $72(70)$ & 12 & 67 & $75(72)$ & 14 \\
Skew_Heap.thy [20] & 16 & 8 & $6(5)$ & 4 & 12 & $8(7)$ & 5 \\
Hash_Code.thy [11] & 16 & 7 & 4 & 4 & 11 & 4 & 7 \\
CoCallGraph.thy [4] & 141 & 88 & $78(71)$ & 29 & 104 & $79(73)$ & 33 \\
Coinductive_Language.thy [28] & 139 & 57 & $69(68)$ & 11 & 106 & $70(69)$ & 43 \\
Context_Free_Grammar.thy [28] & 29 & 26 & 2 & 26 & 29 & 2 & 27 \\
LTL.thy [25] & 97 & 56 & 61 & 15 & 78 & $65(62)$ & 15 \\
HOL/Library/Tree.thy & 124 & 93 & $70(68)$ & 32 & 101 & $73(70)$ & 32 \\
HOL/Library/Tree_Multiset.thy & 8 & 8 & 1 & 7 & 8 & 1 & 7 \\
sum & 801 & 442 & $419(404)$ & 154 & 583 & $437(417)$ & 199 \\
\hline
\end{tabular}

that given 300 seconds of time-out for each proof goal try hard solves 1115 proof goals out of 1526, while sledgehammer found proofs for 901 of them using the same computational resources and re-constructed proofs in Isabelle for 866 of them. This is a 14 percentage point improvement of proof search and a 16 percentage point increase for proof reconstruction. Moreover, 299 goals (20\% of all goals) were solved only by try_hard within 300 seconds. They also show that a longer time-out improves the success ratio of try hard, which is desirable for utilising engineers' downtime.

try_hard is particularly more powerful than sledgehammer at discharging proof obligations that can be nicely handled by the following:

- mathematical induction or co-induction,

- type class mechanism,

- specific procedures implemented as specialised tactics (such as transfer and normalization), or

- general simplification rules (such as field_simps and algebra_simps) .

Furthermore, careful observation of PSL indicates that PSL can handle the so-called "hidden-fact" problem of relevance filter. "Hidden facts" are auxiliary lemmas that are useful to discharge a given proof obligation but hard to pick up for a relevance filter because they are "hidden" in the definition of other

found proofs but sledgehammer did not. POs stands for the number of proof obligations in the theory file. $x(y)$ for SH means sledgehammer found proofs for $x$ proof obligations, out of which it managed to reconstruct proof scripts in Isabelle for $y$ goals. We omit these parentheses when these numbers coincide. Note that all proofs of PSL are checked by Isabelle/HOL. Besides, sledgehammer inside PSL avoids the smt proof method, as this method is not allowed in the Archive of Formal Proofs. 
facts. With PSL, users can write strategies, which first applies rewriting to a given conjecture to reveal these hidden facts to the relevance filter. For example, the following strategy "massages" the given proof obligation before invoking the relevance filter of sledgehammer: Thens [Auto, Repeat (Hammer), IsSolved].

For 3 theories out of 35, try hard discharged fewer proof obligations, even given 300 seconds of time-out. This is due to the fact that PSL uses a slightly restricted version of sledgehammer internally for the sake of the integration with other tools and to avoid the smt method, which is not allowed in the AFP. In these files, sledgehammer can discharge many obligations and other obligations are not particularly suitable for other sub-tools in try_hard. Of course, given high-performance machines, users can run both try_hard and sledgehammer in parallel to maximise the chance of proving conjectures.

\section{Monadic Interpretation of Strategy}

The implementation of the tracing mechanism described in Section 4 is nontrivial: PSL's tracing mechanism has to support arbitrary strategies conforming to its syntax. What is worse, the runtime behaviour of backtracking search is not completely predictable statically since PSL generates tactics at runtime, using information that is not available statically. Moreover, the behaviour of each tactic varies depending on the proof context and proof obligation at hand.

Implementation based on references or pointers is likely to cause code clutter, whereas explicit construction of search tree [16] consumes too much memory space when traversing a large search space. Furthermore, both of these approaches deviate from the standard execution model of Isabelle explained in Section 2, which makes the proof search and the efficient proof script generation less reliable. In this section, we introduce our monadic interpreter for PSL, which yields a modular design and concise implementation of PSL's runtime system.

Monads in Standard ML. A monad with zero and plus is a constructor clas $2^{2}$ with four basic methods. As Isabelle's implementation language, Standard ML, does not natively support constructor classes, we emulated them using its module system [18. Program 1 shows how we represent the type constructor, seq, as an instance of monad with zero and plus.

The body of bind for lazy sequences says that it applies $f$ to all the elements of xs and concatenates all the results into one sequence. Attentive readers might notice that this is equivalent to the behaviour of THEN depicted in Fig. $2 \mathrm{~b}$ and that of Thens shown in Fig. 4. In fact, we can define all of THEN, succeed, fail, and APPEND, using bind, return, mzero, and ++, respectively.

Monadic Interpretation of Strategies. Based on this observation, we formalised PSL's search procedure as a monadic interpretation of strategies, as shown in Program 2, where the type core_strategy stands for the internal representation of strategies. Note that Alt and Or are binary versions of Alts and Ors,

\footnotetext{
2 Constructor classes are a class mechanism on type constructors.
} 

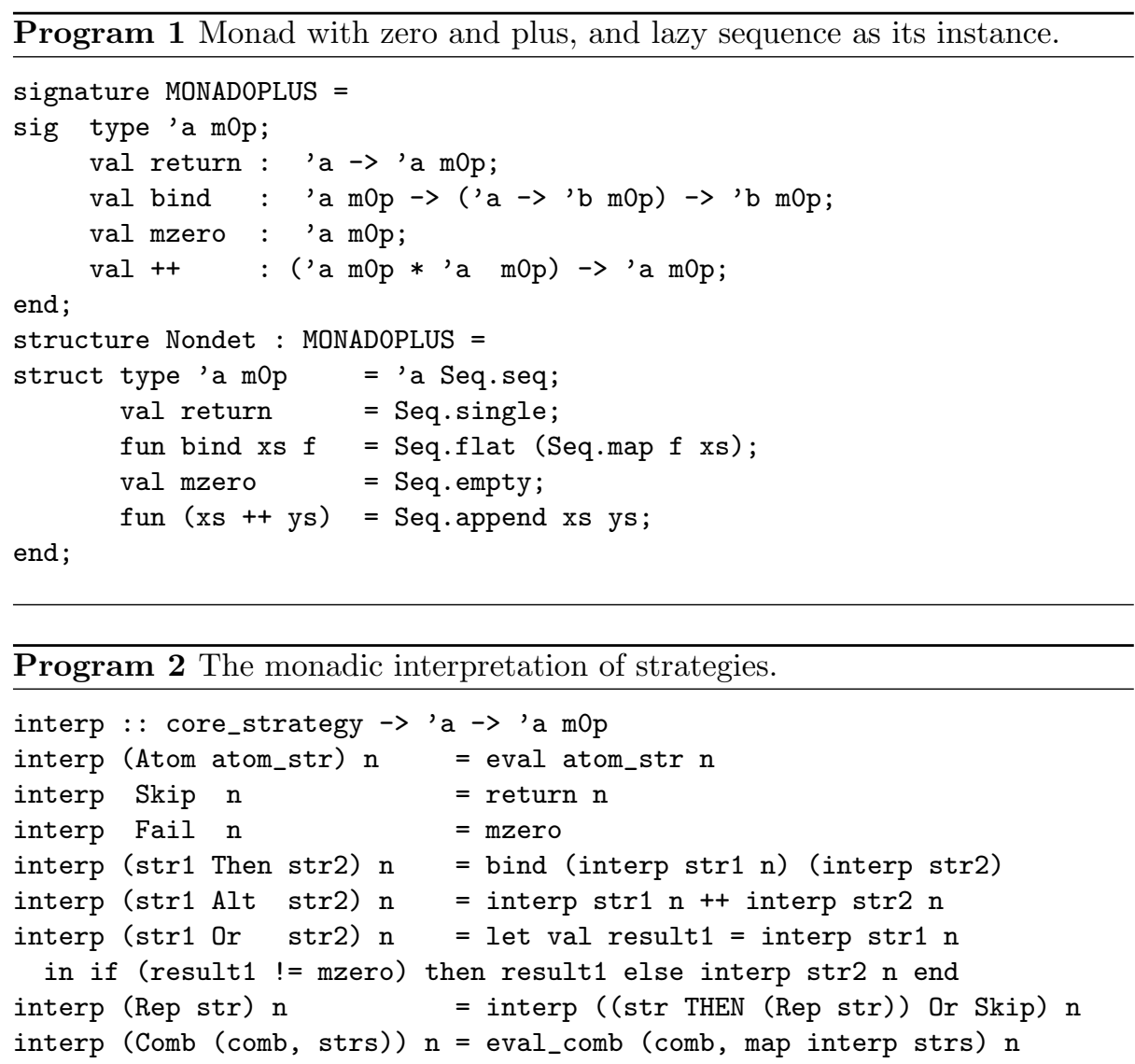

respectively; PSL desugars Alts and Ors into nested Alts and Ors. We could have defined Or as a syntactic sugar using Alt, mzero, Fail, and Skip, as explained by Martin et al. 12]; however, we prefer the less monadic formalisation in Program 2 for better time complexity.

eval handles all the atomic strategies, which correspond to default, dynamic, and special in the surface language. For the dynamic strategies, eval expands them into dynamically generated tactics making use of contextual information from the current proof state. PSL combines these generated tactics either with APPEND or ORELSE, depending on the nature of each tactic. eval_comb handles non-monadic strategy combinators, such as Cut. We defined the body of eval and eval_comb for each atomic strategy and strategy combinator separately using pattern matching. As is obvious in Program 2, interp separates the complexity of compound strategies from that of runtime tactic generation.

Adding Tracing Modularly for Proof Script Generation. We defined interp at the constructor class level, abstracting it from the concrete type of proof state 


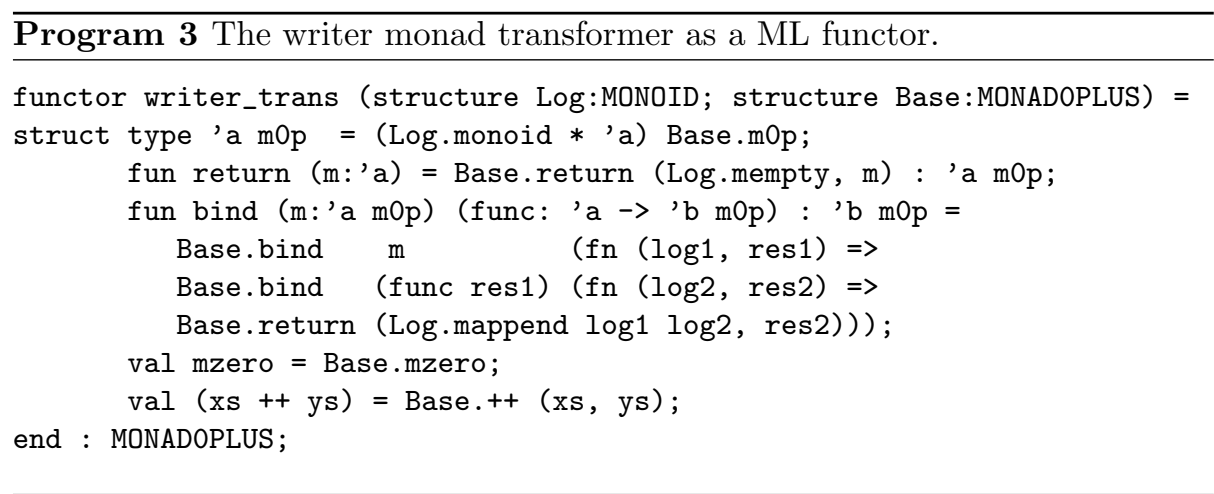

and even from the concrete type constructor. When instantiated with lazy sequence, interp tries to return the first element of the sequence, working as depth-first search. This abstraction provides a clear view of how compound strategies guide proof search while producing tactics at runtime; however, without tracing proof attempts, PSL has to traverse large search spaces every time it checks proofs.

We added the tracing mechanism to interp, combining the non-deterministic monad, Nondet, with the writer monad. To combine multiple monads, we emulate monad transformers using ML functors: Program 3 shows our ML functor, writer_trans, which takes a module of MONADOPLUS, adds the logging mechanism to it, and returns a module equipped with both the capability of the base monad and the logging mechanism of the writer monad. We pass Nondet to writer_trans as the base monad to combine the logging mechanism and the backtracking search based on non-deterministic choice. Observe Program 1, 2 and 3 to see how Alt and Or truncate failed proof attempts while searching for a proof. The returned module is based on a new type constructor, but it is still a member of MONADOPLUS; therefore, we can re-use interp without changing it.

History-Sensitive Tactics using the State Monad Transformer. The flexible runtime interpretation might lead PSL into a non-terminating loop, such as REPEAT succeed. To handle such loops, PSL traverses a search space using iterative deepening depth first search (IDDFS). However, passing around information about depth as an argument of interp as following ${ }^{3}$ quickly impairs its simplicity.

interp ( $\mathrm{t} 1$ CSeq t2) level $\mathrm{n}=$ if level $<1$ then return $\mathrm{n}$ else .. interp ( $t 1 \mathrm{COr}$ t2) level $\mathrm{n}=\ldots$

We implemented IDDFS without code clutter, introducing the idea of a historysensitive tactic: a tactic that takes the log of proof attempts into account. Since the writer monad does not allow us to access the log during the search time, we replaced the writer monad transformer with the state monad transformer,

\footnotetext{
3 level stands for the remaining depth interp can proceed for the current iteration.
} 
with which the runtime keeps the log of proof attempt as the "state" of proof search and access it during search. By measuring the length of "state", interp computes the current depth of proof search at runtime.

The modular design and abstraction discussed above made this replacement possible with little change to the definition of interp: we only need to change the clause for Atom, providing a wrapper function, iddfc, for eval, while other clauses remain intact.

inter (CAtom atom_str) $\mathrm{n}=$ iddfc limit eval atom_str $\mathrm{n}$

iddfc limit first reads the length of "state", which represents the number of edges to the node from the top of the implicit proof search tree. Then, it behaves as fail if the length exceeds limit; if not, it executes eval atom_str n $4^{4}$

\section{Related Work}

ACL2 [10] is a functional programming language and mostly automated firstorder theorem prover, while PSL is embedded in Isabelle/HOL to support higherorder logic. ACL2 is known for the so-called waterfall model, which is essentially repeated application of various heuristics. Its users can guide proof search by supplying arguments called "hints", but the underlining operational procedure of the waterfall model itself is fixed. ACL2 does not produce efficient proof scripts after running the waterfall algorithm.

PVS [23] provides a collection of commands called "strategies". Despite the similarity of the name to PSL, strategies in PVS correspond to tactics in Isabelle. The highest-level strategy in $P V S$, grind, can produce re-runnable proof scripts containing successful proof steps only. However, scripts returned by grind describe steps of much lower level than human engineers would write manually, while PSL's returned scripts are based on tactics engineers use. Furthermore, grind is known to be useful to complete a proof that does not require induction, while try_hard is good at finding proofs involving mathematical induction.

SEPIA [9] is an automated proof generation tool in Coq. Taking existing Coq theories as input, SEPIA first produces proof traces, from which it infers an extended finite state machine. Given a conjecture, SEPIA uses this model to search for its proof. SEPIA's search algorithm is based on the breadth-first search (BFS) to return the shortest proof. PSL can also adopt BFS, as BFS is a special case of IDDFS. However, our experience tells that the search tree tends to be very wide and some tactics, such as induct, need to be followed by other tactics to complete proofs. Therefore, we chose IDDFS for PSL. Both SEPIA and PSL off-load the construction of proof scripts to search and try to reconstruct efficient proof scripts. Compared to SEPIA, PSL allows users to specify their own search strategies to utilize the engineer's intuition, which enables PSL to return incomplete proof scripts, as discussed in Section 4.

Martin et al. first discussed a monadic interpretation of tactics for their language, Angel, in an unpublished draft [13]. We independently developed interp

\footnotetext{
$\overline{4}$ In this sense, we implemented IDDFS as a tactic combinator.
} 
with the features discussed above, lifting the framework from the tactic level to the strategy level to traverse larger search spaces. The two interpreters for different ITPs turned out to be similar to each other, suggesting our approach is not specific to Isabelle but can be used for other ITPs.

Similar to Ltac [6] in Coq, Eisbach [14] is a framework to write proof methods in Isabelle. Proof methods are the Isar syntactic layer of tactics. Eisbach does not generate methods dynamically, trace proof attempts, nor support parallelism natively. Eisbach is good when engineers already know how to prove their conjecture, while try hard is good when they want to find out how to prove it.

IsaPlanner [7] offers a framework for encoding and applying common patterns of reasoning in Isabelle, following the style of proof planning [5. IsaPlanner addresses the performance issue by memoization technique, on the other hand try_hard strips off backtracked steps while searching for a proof, which Isabelle can check later without try_hard. While IsaPlanner works on its own data structure reasoning state, try_hard managed to minimize the deviation from Isabelle's standard execution model using constructor classes.

\section{Conclusions}

Proof automation in higher-order logic is a hard problem: every existing tool has its own limitation. PSL attacks this problem, allowing us to exploit both the engineer's intuition and various automatic tools. The simplicity of the design is our intentional choice: we reduced the process of interactive proof development to the well-known dynamic tree search problem and added new features (efficientproof script generation and IDDFS) by safely abstracting the original execution model and employing commonly used techniques (monad transformers).

We claim that our approach enjoys significant advantages. Despite the simplicity of the design, our evaluations indicate that PSL reduces the labour cost of ITP significantly. The conservative extension to the original model lowers the learning barrier of PSL and makes our proof-script generation reliable by minimising the deviation. The meta-tool approach makes the generated proof-script independent of PSL, separating the maintenance of proof scripts from that of PSL; furthermore, by providing a common framework for various tools we supplement one tool's weakness (e.g. induction for sledgehammer) with other tools' strength (e.g. the induct tactic), while enhancing their capabilities with runtime tactic generation. The parallel combinators transforms the conventionally labour-intensive interactive theorem proving to embarrassingly parallel problems. The abstraction to the constructor class and reduction to the tree search problem make our ideas transferable: other ITPs, such as Lean and Coq, handle inter-tactic backtracking, which is best represented in terms of MONADOPLUS.

Acknowledgements We thank Jasmin C. Blanchette for his extensive comments that improved the evaluation of try_hard. Pang Luo helped us for the evaluation. Leonardo de Moura, Daniel Matichuk, Kai Engelhardt, and Gerwin Klein provided valuable comments on an early draft of this paper. 


\section{References}

1. Blanchette, J., Fleury, M., Wand, D.: Concrete Semantics with Isabelle/HOL (2015), http://people.mpi-inf.mpg.de/ jblanche/cswi/

2. Blanchette, J.C., Kaliszyk, C., Paulson, L.C., Urban, J.: Hammering towards QED. J. Formalized Reasoning 9(1), 101-148 (2016), http://dx.doi.org/10. 6092/issn.1972-5787/4593

3. Böhme, S., Nipkow, T.: Sledgehammer: Judgement day. In: Giesl, J., Hähnle, R. (eds.) Automated Reasoning, 5th International Joint Conference, IJCAR 2010, Edinburgh, UK, July 16-19, 2010. Proceedings. Lecture Notes in Computer Science, vol. 6173, pp. 107-121. Springer (2010), http://dx.doi.org/10.1007/ 978-3-642-14203-1_9

4. Breitner, J.: The safety of call arity. Archive of Formal Proofs (Feb 2015), http: //isa-afp.org/entries/Call_Arity.shtml, Formal proof development

5. Bundy, A.: The use of explicit plans to guide inductive proofs. In: Lusk, E.L., Overbeek, R.A. (eds.) 9th International Conference on Automated Deduction, Argonne, Illinois, USA, May 23-26, 1988, Proceedings. Lecture Notes in Computer Science, vol. 310, pp. 111-120. Springer (1988), http://dx.doi.org/10.1007/BFb0012826

6. Delahaye, D.: A tactic language for the system coq. In: Parigot, M., Voronkov, A. (eds.) Logic for Programming and Automated Reasoning, 7th International Conference, LPAR 2000, Reunion Island, France, November 11-12, 2000, Proceedings. Lecture Notes in Computer Science, vol. 1955, pp. 85-95. Springer (2000), http://dx.doi.org/10.1007/3-540-44404-1_7

7. Dixon, L., Fleuriot, J.D.: IsaPlanner: A prototype proof planner in Isabelle. In: Baader, F. (ed.) Automated Deduction - CADE-19, 19th International Conference on Automated Deduction Miami Beach, FL, USA, July 28 - August 2, 2003, Proceedings. Lecture Notes in Computer Science, vol. 2741, pp. 279-283. Springer (2003), http://dx.doi.org/10.1007/978-3-540-45085-6_22

8. Felty, A.P., Middeldorp, A. (eds.): Automated Deduction - CADE-25 - 25th International Conference on Automated Deduction, Berlin, Germany, August 1-7, 2015, Proceedings, Lecture Notes in Computer Science, vol. 9195. Springer (2015), http://dx.doi.org/10.1007/978-3-319-21401-6

9. Gransden, T., Walkinshaw, N., Raman, R.: SEPIA: search for proofs using inferred automata. In: Felty and Middeldorp 8], pp. 246-255, http://dx.doi.org/ 10.1007/978-3-319-21401-6_16

10. Kaufmann, M., Moore, J.S., Manolios, P.: Computer-Aided Reasoning: An Approach. Kluwer Academic Publishers, Norwell, MA, USA (2000)

11. Lammich, P.: Collections framework. Archive of Formal Proofs (Nov 2009), http: //isa-afp.org/entries/Collections.shtml, Formal proof development

12. Martin, A.P., Gardiner, P.H.B., Woodcock, J.: A tactic calculus-abridged version. Formal Asp. Comput. 8(4), 479-489 (1996), http://dx.doi.org/10.1007/ BF01213535

13. Martin, A., Gibbons, J.: A monadic interpretation of tactics (2002)

14. Matichuk, D., Wenzel, M., Murray, T.C.: An Isabelle proof method language. In: Klein, G., Gamboa, R. (eds.) Interactive Theorem Proving - 5th International Conference, ITP 2014, Held as Part of the Vienna Summer of Logic, VSL 2014, Vienna, Austria, July 14-17, 2014. Proceedings. Lecture Notes in Computer Science, vol. 8558, pp. 390-405. Springer (2014), http://dx.doi.org/10.1007/ 978-3-319-08970-6_25 
15. de Moura, L.M., Kong, S., Avigad, J., van Doorn, F., von Raumer, J.: The lean theorem prover (system description). In: Felty and Middeldorp [8], pp. 378-388, http://dx.doi.org/10.1007/978-3-319-21401-6_26

16. Nagashima, Y.: Keep failed proof attempts in memory. In: Isabelle Workshop 2016. Nancy, France (aug 2016)

17. Nagashima, Y.: PSL (2016), https://github.com/data61/PSL

18. Nagashima, Y., O'Connor, L.: Close encounters of the higher kind - emulating constructor classes in standard ML (sep 2016)

19. Nipkow, T.: List index. Archive of Formal Proofs (Feb 2010), http://isa-afp. org/entries/List-Index.shtml, Formal proof development

20. Nipkow, T.: Skew heap. Archive of Formal Proofs (Aug 2014), http://isa-afp. org/entries/Skew_Heap.shtml, Formal proof development

21. Nipkow, T., Paulson, L.C., Wenzel, M.: Isabelle/HOL - A Proof Assistant for Higher-Order Logic, Lecture Notes in Computer Science, vol. 2283. Springer (2002), http://dx.doi.org/10.1007/3-540-45949-9

22. Nishihara, T., Minamide, Y.: Depth first search. Archive of Formal Proofs (Jun 2004), http://isa-afp.org/entries/Depth-First-Search.shtml, Formal proof development

23. Owre, S., Rushby, J.M., Shankar, N.: Pvs: A prototype verification system. In: Proceedings of the 11th International Conference on Automated Deduction: $\mathrm{Au}-$ tomated Deduction. pp. 748-752. CADE-11, Springer-Verlag, London, UK, UK (1992), http://dl.acm.org/citation. cfm?id=648230.752639

24. Paulson, L.C.: The foundation of a generic theorem prover. CoRR cs.LO/9301105 (1993), http://arxiv.org/abs/cs.LO/9301105

25. Sickert, S.: Linear temporal logic. Archive of Formal Proofs (Mar 2016), http: //isa-afp.org/entries/LTL.shtml, Formal proof development

26. Sternagel, C.: Efficient mergesort. Archive of Formal Proofs (Nov 2011), http:// isa-afp.org/entries/Efficient-Mergesort.shtml, Formal proof development

27. development team, T.C.: The coq proof assistant reference manual (2009)

28. Traytel, D.: A codatatype of formal languages. Archive of Formal Proofs (Nov 2013), http://isa-afp.org/entries/Coinductive_Languages.shtml, Formal proof development

29. Wadler, P.: How to replace failure by a list of successes: A method for exception handling, backtracking, and pattern matching in lazy functional languages. In: Jouannaud, J. (ed.) Functional Programming Languages and Computer Architecture, FPCA 1985, Nancy, France, September 16-19, 1985, Proceedings. Lecture Notes in Computer Science, vol. 201, pp. 113-128. Springer (1985), http://dx.doi.org/10.1007/3-540-15975-4_33 


\section{A Appendix: the Default Strategy, try hard}

The following is the definition of try hard. It starts with simple sub-strategies and gradually proceeds to more involved sub-strategies. Note that try_hard is just one default strategy: we provided PSL as a language, so that users can encode their intuitions as strategies.

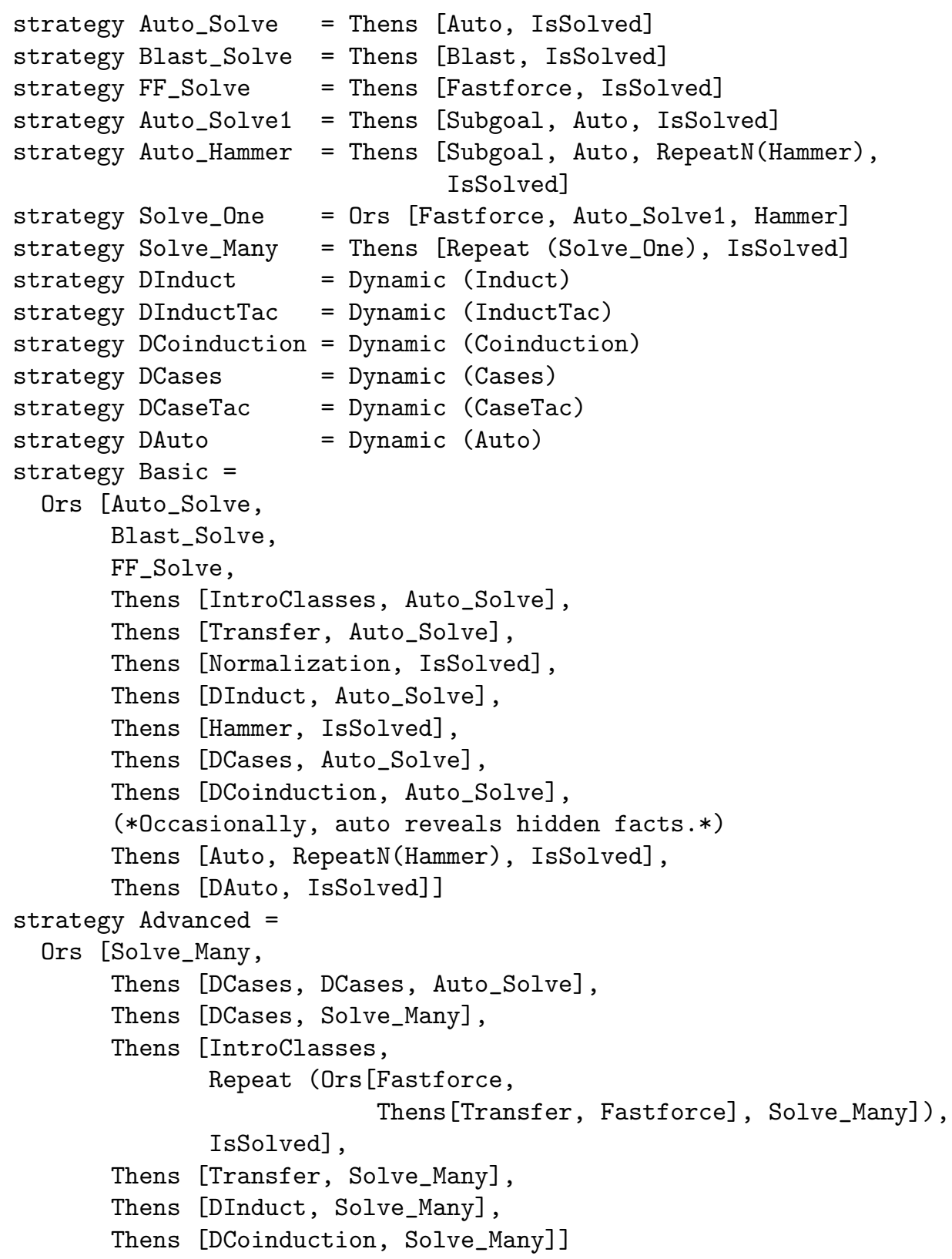




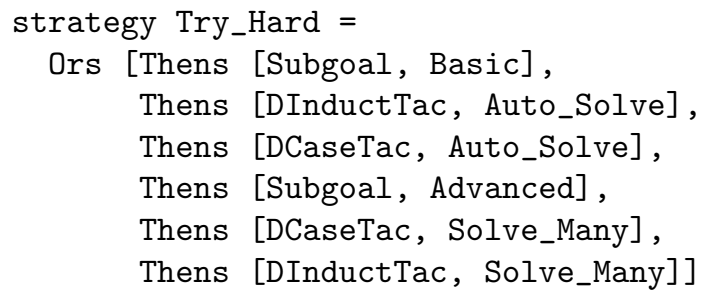

\section{B Appendix: Details of the Evaluation}

All evaluations were conducted on a Linux machine with Intel (R) Core (TM) i7-600@3.40GHz and 32 GB memory. For both tools, we set the time-out of proof search to 30 and 300 seconds for each proof obligation.

Prior to the evaluation, the relevance filter of sledgehammer was trained on 27,041 facts and 18,695 non-trivial Isar proofs from the background libraries imported by theories under evaluation for both tools. Furthermore, we forbid sledgehammer inside PSL from using the smt method for proof reconstruction, since the AFP does not permit this method.

Note that try hard does not use parallel strategy combinators which exploit parallelism. The evaluation tool does not allow try_hard to use multiple threads either. Therefore, given the same time-out, try hard and sledgehammer enjoy the same amount of computational resources, assuring the fairness of the evaluation results.

We provide the evaluation results in the following website for the purpose of reviewing:

- http://ts.data61.csiro.au/Private/downloads/cade26_results/

\section{Appendix: Note on Examples.}

The examples of efficient-proof-script generation in Section 4 have been demonstrated on machines at Data61. Depending on the hardware conditions and the prior training of sledgehammer's relevance filter, one may obtain different results. One can configure the number of threads for proof search by inserting the following ML code snippet inside the ongoing Isabelle proof script:

ML $\{*$ Multithreading.max_threads_update $56 *\}$

ML $\{*$ Goal.parallel_proofs : $=0 *\}$ 